Pacific Journal of Mathematics

REPRESENTATION THEORY OF ALMOST CONNECTED 


\title{
REPRESENTATION THEORY OF ALMOST CONNECTED GROUPS
}

\author{
RONALD LIPSMAN
}

Let $G$ be a locally compact group and $G_{0}$ its connected component of the identity. If $G / G_{0}$ is compact, then $G$ is a projective limit of Lie groups. In fact, there exist arbitrarily small compact normal subgroups $H \cong G$ such that $G / H$ is a Lie group. Suppose $H$ is such a compact, co-Lie subgroup of $G$. Then any unitary representation of $G / H$ can be lifted to $G$ in a natural way. Conversely, given a unitary representation $\pi$ of $G$, one may ask whether it really lives on a Lie factor-that is, does there always exist a compact normal subgroup $H \subseteq G$ such that $G / H$ is a Lie group and $\pi(h), h \in H$, is the identity operator? In this paper it is shown that this is indeed the case whenever $\pi$ is irreducible (or more generally whenever $\pi$ is factorial). The dual space $\hat{G}$ (=equivalence classes of irreducible unitary representations) is then realized as an inductive limit of the dual spaces of Lie groups. This inductive limit is first cast in a topological setting (using the dual topology on $\hat{G}$ ); and then, when $G$ is also unimodular and type $I$, one obtains a measure-theoretic interpretation of the inductive limit (using the Plancherel measure). One application of these results is the fact that an almost connected group whose solvable radical is actually nilpotent must be a type I group.

1. Introduction. Let $G$ be a locally compact group with left Haar measure $d g$. Denote by $\operatorname{Irr}(G)$ the collection of irreducible unitary representations of $G$, and by $\hat{G}$ the quotient space obtained from $\operatorname{Irr}(G)$ by the relation of unitary equivalence. When there is no possibility of confusion we shall fail to distinguish between a reperesentation $\pi \in \operatorname{Irr}(G)$ and its class $\{\pi\} \in \hat{G}$. It is possible to endow $\hat{G}$ with a locally compact (generally, non-Hausdorff) topology [6]; and with that topology $\hat{G}$ is called the dual space of $G$. Suppose in addition that $G$ is unimodular and type I; then there is a unique positive Radon measure $\mu_{G}$ on $\hat{G}$ (called the Plancherel measure - see [3]) such that

$$
\int_{G}|f(g)|^{2} d g=\int_{\hat{G}} \operatorname{Tr}\left[\pi(f)^{*} \pi(f)\right] d \mu_{G}(\pi), \quad f \in L_{1}(G) \cap L_{2}(G),
$$

where $\pi(f)=\int_{G} f(g) \pi(g) d g$.

Now by an almost connected group we mean a locally compact group $G$ such that $G / G_{0}$ is compact, $G_{0}=$ the connected component of the identity. It is well-known that such groups are projective limits 
of Lie groups; more precisely, given any neighborhood $\mathscr{Z}$ of the identity in $G$, there exists a compact normal subgroup $H \cong \mathscr{Q}$ such that $G / H$ is a Lie group [14, p. 175]. Let us denote $\mathscr{L}=\mathscr{L}(G)=\{H \subseteq$ $G$ : $H$ compact normal in $G$ and $G / H$ is a Lie group\}. Then $\mathscr{C}$ is a directed set (since $H_{1}, H_{2} \in \mathscr{L} \Rightarrow H_{1} \cap H_{2} \in \mathscr{P}$ [14, p. 177]) and

$$
G=\lim _{I I \in \mathscr{C}} G / H \text {. }
$$

The main idea that will be discussed in this paper is a representation-theoretic dual of equation (1.2). More explicitly, let $H \in \mathscr{L}(G)$ and let $j_{H}: G \rightarrow G / H$ be the canonical projection. Then there is a "dual" or "adjoint" map $\hat{j}_{H}: \widehat{G / H} \rightarrow \widehat{G}$ given by $\left(\hat{j}_{I I} \lambda\right)(g)=\lambda\left(j_{H}(g)\right)$, $g \in G, \lambda \in \widehat{G / H}$. We denote by $\widehat{G}_{H}$ the image of $\widehat{G / H}$ in $\hat{G}$ under the injective map $\hat{j}_{I I}$. The dual equation we seek to establish is

$$
\widehat{G}=\lim _{I I \in \mathscr{L}} \hat{G}_{I I}
$$

In $\S 2$ we demonstrate the natural set-theoretic interpretation of (1.3), namely we show that $\hat{G}=\bigcup_{H \in \mathscr{L}} \hat{G}_{H}$. Then in $\S 3$ we extend this result from irreducible to factorial representations of $G$. We show how this leads to "smoothness" criteria for $\hat{G}$ in terms of those for $\widehat{G / H}, H \in \mathscr{L}$. These criteria are used in $\S 4$ to deduce that all groups of a certain important kind are type I. Specifically, if $G$ is almost connected then it contains a unique radical $R=R(G)=$ the maximal connected solvable normal subgroup. We prove that any group whose radical is nilpotent is a type I group. We further consider a somewhat smaller class of groups (which we call traceable) for which there is an adequate "character theory." We show that all semisimple (i.e., $R(G)$ trivial) and nilpotent groups are traceable. Next in $\S 5$, we make (1.3) precise in first a topological, and then a measure-theoretic fashion. In fact we shall show that $\hat{G}$ is a topological inductive limit (in a rather strong sense) of the topological spaces $\widehat{G / H}$; and that when $G$ is unimodular and type I, the Plancherel measure $\mu_{G}$ is an inductive limit of the measures $\mu_{G \mid I I}$. Finally in an appendix we give a brief survey of how the structure and representation theory of connected semisimple Lie groups (due mostly to Harish-Chandra) can be extended to arbitrary connected semisimple groups.

Before beginning we establish some notation which-together with that set down above-will be standard in the following. $G$ will denote a locally compact group; usually $G / G_{0}$ will be compact. $C_{0}(G)$ stands for the continuous functions of compact support, and if $G$ is a Lie group $C^{\infty}(G)=$ the infinitely differentiable functions. We write $\mathscr{D}(G)$ for $C_{0}(G) \cap C^{\infty}(G)$ with its usual Schwartz topology. If $\pi$ is a unitary representation of $G$, we denote by $\mathscr{C}(\pi)$ the Hilbert space 
on which $\pi$ acts. We set $\mathscr{R}(\pi)=$ the $W^{*}$-algebra of operators generated by $\{\pi(g): g \in G\}$. Then $\operatorname{Fac}(G)=\{\pi: \mathscr{R}(\pi)$ is a factor $\}$. For two representations $\pi_{1}, \pi_{2}$, we write $\pi_{1} \cong \pi_{2}$ to mean unitary eqivalence and $\pi_{1} \approx \pi_{2}$ for quasi-equivalence. If $\mathscr{T}^{\prime}$ is a subset of a Hilbert space $\mathscr{H}, \overline{\mathrm{sp}}\{\mathscr{K}\}$ will denote the smallest closed subspace of $\mathscr{\mathscr { C }}$ containing $\mathscr{K}$. Finally, $I$ will always denote the identity operator on $\mathscr{C}$, as well as the identity representation on $\mathscr{\mathscr { C }}$ of whatever group $G$ is under consideration.

2. The main result. We show first that $\hat{G}=\bigcup_{I I \in \mathscr{L}} \hat{G}_{I I}$. This amounts to the following. Given $\pi \in \operatorname{Irr}(G)$, we have to produce an $H \in \mathscr{L}(G)$ such that $\left.\pi\right|_{I I}=I$. Then $\lambda\left(j_{I I}(g)\right)=\pi(g), g \in G$, is welldefined, $\lambda \in \operatorname{Irr}(G / H)$, the class of $\lambda$ depends only on the class of $\pi$, and $\{\pi\}=\hat{j}_{I I}\{\lambda\}$.

THEOREM 2.1. Let $G$ be a locally compact almost connected group. Then for any $\pi \in \operatorname{Irr}(G)$, there exists a compact normal subgroup $H \leqq G$ such that $\left.\pi\right|_{H}=I$ and $G / H$ is a Lie group.

We give the proof in several steps. The first is an easy

LEMMA 2.2. Let $\lambda$ be a unitary representation of a compact group $H$ on a Hilbert space $\mathscr{H}$. Suppose that $\left\{\mathscr{C}_{\alpha}\right\}_{\alpha \in \Omega}$ is a family of (finitedimensional) invariant irreducible subspaces of $\mathscr{H}$. Denote by $\lambda_{\alpha}$ the restriction of $\lambda$ to $\mathscr{H}_{\alpha}$. Assume that $\overline{\mathrm{sp}}\left\{\xi: \xi \in \mathscr{H}_{a}, \alpha \in \Omega\right\}=\mathscr{H}_{\text {. Then }}$ any irreducible subrepresentation of $\lambda$ is unitarily equivalent to one of the $\lambda_{\alpha}$.

Proof. Let . $\%$ be any invariant irreducible subspace, $\sigma$ the resulting representation of $H$. Let $\alpha \in \Omega$ be arbitrary, and set $P_{\kappa}=$ the orthogonal projection of $\mathscr{H}$ onto $\mathscr{C}_{\alpha}$. Since $\mathscr{C}_{\alpha}$ is invariant, $P_{\alpha} \lambda(h)=\lambda(h) P_{\alpha}$. But then for $\xi \in \mathscr{K}$, we have $\lambda_{\alpha}(h) P_{\alpha} \xi=\lambda(h) P_{\alpha} \xi=$ $P_{\alpha} \lambda(h) \xi=P_{\alpha} \sigma(h) \xi$. Since $\mathscr{H}_{\alpha}$ and $\mathscr{\mathscr { C }}$ are irreducible, the Schur Theorem $[4, \S 2.3 .4]$ guarantees that either $P_{\alpha}(\mathscr{K})=\{0\}$-that is, $\mathscr{K}$ and $\mathscr{H}_{\alpha}$ are orthogonal-or $\sigma$ and $\lambda_{\alpha}$ are unitarily equivalent. But since the collection $\left\{\mathscr{C}_{\alpha}: \alpha \in \Omega\right\}$ spans $\mathscr{H}$, it is impossible for $\mathscr{\mathscr { C }}$ to be orthogonal to all the $\mathscr{K}_{\alpha}$.

In our next lemma, we use the following idea. Let $G$ be a locally compact group and $H \leqq G$ a closed normal subgroup. Then $G$ acts as a transformation group of the topological space $\hat{H}$ by $(g, \lambda) \rightarrow$ $g \cdot \lambda,(g \cdot \lambda)(h)=\lambda\left(g^{-1} h g\right), h \in H, g \in G, \lambda \in \hat{H}$. This action is in fact jointly continuous [7, Lemma 1]; but we shall need only the (easily established) continuity of $g \rightarrow g \cdot \lambda, \lambda$ fixed, in what follows. 
LEMMA 2.3. Let $G$ be a locally compact almost connected group, $H \leqq G$ a compact normal subgroup, $\pi \in \operatorname{Irr}(G)$. Then we can find subspaces $\mathscr{H}_{\alpha}, \alpha \in \Omega$, of $\mathscr{H}=\mathscr{H}(\pi)$, invariant and irreducible under $\left.\pi\right|_{H}$, such that:

(i) $\overline{\operatorname{sp}}\left\{\mathscr{H}_{\alpha}: \alpha \in \Omega\right\}=\mathscr{H}$;

(ii) if $\lambda_{\alpha}$ denotes the representation $\left.\pi\right|_{H}$ in the subspace $\mathscr{C}_{\alpha}$, and $\left\{\lambda_{\alpha}\right\}$ its class in $\hat{H}$, then $\bigcup_{\alpha \in \Omega}\left\{\lambda_{\alpha}\right\}$ is a finite subset of $\hat{H}$.

Proof. Since $H$ is compact, we can always find a nonzero subspace of $\mathscr{L}$, say $\mathscr{K}$, invariant and irreducible under $\left.\pi\right|_{H}$. Let $\lambda$ denote the representation $\left.\pi\right|_{H H}$ in $\mathscr{K}$. Now if $\xi \in \mathscr{K}, g \in G, h \in H$, then $\pi(h) \pi(g) \xi=\pi(g) \pi\left(g^{-1} h g\right) \xi \in \pi(g) \mathscr{K}$, i.e., $\pi(g) \mathscr{K}$ is also invariant under $\left.\pi\right|_{H}$. Let $\lambda_{g}$ denote the representation $\left.\pi\right|_{H}$ in $\pi(g) \mathscr{K}$. Note that it follows from the irreducibility of $\pi$ that $\overline{\mathrm{sp}}\{\pi(g) \mathscr{K}: g \in G\}=\mathscr{H}$.

Now consider the continuous map $g \rightarrow\{g \cdot \lambda\}$ of $G$ into $\hat{H}$. Since $H$ is compact, the space $\hat{H}$ is actually discrete [4, p. 322]. Therefore the inverse image $G_{\lambda}$ of $\{\lambda\}, G_{\lambda}=\{g \in G: g \cdot \lambda \cong \lambda\}$, is an open subgroup of $G$. In particular $G_{0} \subseteq G_{2}$. Then $G / G_{2}$ is both compact and discrete-so it's finite. Choose a set of representatives $g_{1}, \cdots, g_{n}$ for the left cosets of $G_{\lambda}$ in $G$.

It is a formality to check that $\xi \rightarrow \pi(g) \xi, \mathscr{K} \rightarrow \pi(g) \mathscr{K}$, is a unitary intertwining operator for $g \cdot \lambda$ and $\lambda_{g}$. Therefore if $g=g_{i} g_{\lambda}, 1 \leqq i \leqq n$, $g_{\lambda} \in G_{\lambda}$, the representation $\lambda_{g}$ is equivalent to $\left(g_{i} g_{\lambda}\right) \cdot \lambda=g_{i} \cdot\left(g_{\lambda} \cdot \lambda\right) \cong g_{i} \cdot \lambda$. Thus we may take for our subspaces the collection $\{\pi(g) \mathscr{K}: g \in G\}$. We have already seen that they satisfy (i); condition (ii) follows from $\bigcup_{g \in G}\left\{\lambda_{g}\right\}=\bigcup_{i=1}^{n}\left\{g_{i} \cdot \lambda\right\}$.

This completes the preliminary stages. We go on now to the main argument.

Proof of Theorem 2.1. Let $\pi \in \operatorname{Irr}(G)$ be given. Choose any $K \in$ $\mathscr{L}(G)$. Of course $\left.\pi\right|_{K}$ is a direct sum of irreducible representations of $K$. It follows from Lemmas 2.2 and 2.3 that there exist elements $\left\{\lambda_{i}\right\} \in \hat{K}, 1 \leqq i \leqq n$, and $K$-invariant subspaces $\mathscr{H}_{1}, \cdots, \mathscr{C}_{n}$ of $\mathscr{\mathscr { C }}=$ $\mathscr{H}(\pi)$ such that $\mathscr{H}=\bigoplus_{i=1}^{n} \mathscr{H}_{i}$ and every irreducible subrepresentation of $\left.\pi\right|_{K}$ in $\mathscr{H}_{i}$ is equivalent to $\lambda_{i}$.

But $\lambda_{i}$ is finite-dimensional, say $\operatorname{dim} \lambda_{i}=m_{i}$. Thus $\lambda_{i}$ is actually a continuous homomorphism of $K$ into the compact Lie group $U\left(m_{i}\right)$ of unitary $m_{i} \times m_{i}$ matrices. Letting $K_{i}=\operatorname{kernel} \lambda_{i}$, we see that $K / K_{i}$ is a compact Lie group.

Clearly $\left.\pi\right|_{K_{i}}=I$ on the subspace $\mathscr{H}_{i}$. But $K_{i}$ may not be normal in $G$, so we have to shrink it somewhat. Set $G_{i}=\left\{g \in G: g \cdot \lambda_{i} \cong \lambda_{i}\right\}$. Exactly as in the proof of Lemma 2.3, one proves that $G_{i}$ is an open subgroup of finite index in $G$. Moreover $K_{i}$ is normal in $G_{i}$. Indeed 
let $g \in G_{i}, k \in K_{i}$. Then $\lambda_{i}\left(g^{-1} k g\right)=\left(g \cdot \lambda_{i}\right)(k)=I$, since $g \cdot \lambda_{i} \cong \lambda_{i}$ and $K_{i}$ depends only on the class of $\left\{\lambda_{i}\right\}$.

Set $K_{i}^{\prime}=\bigcap_{g \in G} g K_{i} g^{-1}$, a compact normal subgroup of $G$. Let $g_{1}, \cdots, g_{p_{i}}$ denote a set of representatives for $G / G_{i}$. Then, since $G_{i}$ normalizes $K_{i}$, it is a simple matter to verify that $K_{i}^{\prime}=\bigcap_{j=1}^{p_{i}} g_{j} K_{i} g_{j}^{-1}$. But $K / K_{i}$ is a Lie group and isomorphic to $g_{j} K g_{j}^{-1} / g_{j} K_{i} g_{j}^{-1}=K / g_{j} K_{i} g_{j}^{-1}$. Since the intersection is finite, it follows [14, p. 177] that $K / K_{i}^{\prime}$ is also a Lie group. But the fact that $G / K$ and $K / K_{i}^{\prime}$ are both Lie groups guarantees that $G / K_{i}^{\prime}$ is actually a Lie group [11, Theorem 7].

Thus for each $i, 1 \leqq i \leqq n$, we have found $K_{i}^{\prime}$, compact normal in $G, G / K_{i}^{\prime}$ a Lie group, and $\left.\pi\right|_{K_{i}}=I$ on $\mathscr{H}_{i}$. Let $H=\bigcap_{i=1}^{n} K_{i}^{\prime}$. Then $H \in \mathscr{L}(G)$ and $\left.\pi\right|_{H}=I$.

3. Smoothness in the dual. There are varying degrees of smoothness (mainly separation properties) that the dual space $\hat{G}$ may possess. $\hat{G}$ Hausdorff is a strong imposition on $G$. It appears to force $G$ to be some kind of combination of compact and abelian groups although the precise characterization is not known yet. However, we can ask for a little less and, as we shall see, there are large collections of groups which are "type I" or even "CCR." We show in this section that $\hat{G}$ has either of the latter two properties if and only if each $\widehat{G / H}$ does also. First we need the following generalization of Theorem 2.1.

THEOREM 3.1. Let $G$ be a locally compact almost connected group. Then for any $\pi \in \mathrm{Fac}(G)$, there exists a compact normal subgroup $H \subseteq G$ such that $\left.\pi\right|_{H}=I$ and $G / H$ is a Lie group.

Proof. Let $K \in \mathscr{L}(G)$. Apply the argument used in the proof of Lemma 2.3 to $\left.\pi\right|_{K}$. If we let $\mathscr{K}$ be any nonzero subspace of $\mathscr{H}=\mathscr{C}(\pi)$, invariant and irreducible under $\left.\pi\right|_{K}$, then on the $G$ invariant subspace $\mathscr{K}^{\prime}=\overline{\mathrm{sp}}\{\pi(g) \mathscr{L}: g \in G\}$, the representation $\left.\pi\right|_{K}$ breaks up into a direct sum $\bigoplus_{i=1}^{n} c(i) \lambda_{i}$, for some $\lambda_{i} \in \hat{K}$, and some cardinal numbers $c(i)$. (Note the only place we used the irreducibility of $\pi$ in Lemma 2.3 was to conclude that $\mathscr{C}^{\prime}=\mathscr{C}$.) Let $\pi^{\prime}=\pi$ acting on $\mathscr{C}^{\prime}$. Then employing the reasoning in the proof of Theorem 2.1, we conclude that there is $H \subseteq K$, a compact normal subgroup of $G$, such that $G / H$ is a Lie group and $\left.\pi^{\prime}\right|_{H}=I$.

But $\pi$ is a factorial representation. Therefore it is quasi-equivalent to any subrepresentation, $\pi \approx \pi^{\prime}[4$, p. 106]. Hence there exist cardinals $\alpha, \beta$ such that $\alpha \pi \cong \beta \pi^{\prime}\left[4\right.$, p. 104]. Certainly $\left.\pi^{\prime}\right|_{H}=I \Rightarrow$ $\left.\beta \pi^{\prime}\right|_{H}=\left.I \Rightarrow \alpha \pi\right|_{H}=I$. We can realize $\mathscr{\mathscr { C }}(\alpha \pi)=\mathscr{H}(\pi) \otimes C^{\alpha},(\alpha \pi)(x)=$ $\pi(x) \otimes I$, and so it follows that $\left.\pi\right|_{H}=I$ as well. This completes the 
proof.

Before stating our next theorem we recall some definitions. $G$ is called CCR if for every $\pi \in \operatorname{Irr}(G)$ and $f \in L_{1}(G)$, the operator $\pi(f)$ is compact. $G$ is called type I if for every unitary representation $\pi$ of $G, \mathscr{R}(\pi)$ is a type I $W^{*}$-algebra [4, pp. 338-9]. Of course CCR $\Rightarrow$ type I [4, p. 87], but not conversely. It has long been known that for separable groups, $G$ is type $I \Leftrightarrow$ for every $\pi \in \mathrm{Fac}(G), \mathscr{R}(\pi)$ is type I. A more recent result of Sakai [16, Theorem 2] shows that this is true for non-separable groups as well. (Sakai's theorem also shows that the collection of type I groups coincides with the collection of GCR groups [4, $\S 4.3 .1$ and $\S 13.9 .4]$, again whether $G$ is separable or not.)

THEOREM 3.2. Let $G$ be a locally compact almost connected group. Then $G$ is type $I$ (respectively $C C R$ ) if and only if $G / H$ is type $I$ (respectively $C C R$ ) for every $H \in \mathscr{L}(G)$.

Proof. First suppose $G$ is type I. Let $H \in \mathscr{L}$ and $\pi_{1} \in \mathrm{Fac}(G / H)$. Set $\pi(g)=\pi_{1}\left(j_{H}(g)\right), g \in G$. It is clear that $\mathscr{R}(\pi)=\mathscr{R}\left(\pi_{1}\right)$. Therefore $\pi \in \operatorname{Fac}(G)$ and $\mathscr{R}(\pi)=\mathscr{R}\left(\pi_{1}\right)$ is type I. It follows that $G / H$ is a type I group.

Conversely suppose $G / H$ is type I for all $H \in \mathscr{L}$. It follows immediately from Theorem 3.1 that for every $\pi \in \mathrm{Fac}(G), \mathscr{R}(\pi)$ is type I. So $G$ is a type I group.

Next suppose $G$ is CCR. Let $H \in \mathscr{L}$ and $\pi_{1} \in \operatorname{Irr}(G / H)$. Again setting $\pi(g)=\pi_{1}\left(j_{H}(g)\right), g \in G$, we obtain a representation $\pi \in \operatorname{Irr}(G)$. Let $F \in L_{1}(G / H)$ be arbitrary. Then choose $f \in L_{1}(G)$ so that $F\left(j_{I I}(g)\right)=$ $\int_{I I} f(g h) d h$. This choice is possible since $f \rightarrow F$ is a (bounded) linear map of $L_{1}(G)$ onto $L_{1}(G / H)$. Then we compute $\pi(f)=\int f^{\prime}(g) \pi(g) d g=$ $\int_{G \mid I I} \int_{H} f(g h) \pi(g h) d h d \bar{g}=\int_{G / I I} F(\bar{g}) \pi_{1}(\bar{g}) d \bar{g}=\pi_{1}(F)$. Since $G$ is CCR, it follows that $\pi_{1}\left(F^{\prime}\right)$ is a compact operator $\Rightarrow G / H$ is also CCR.

Finally, let $G / H$ be CCR for all $H \in \mathscr{L}$. Then for any $\pi \in \operatorname{Irr}(G)$, there exists $H \in \mathscr{C}$ such that $\pi(g)=\pi_{1}\left(j_{H}(g)\right), g \in G$, for some $\pi_{1} \in$ $\operatorname{Irr}(G / H)$. Let $f \in L_{1}(G)$. Then a computation similar to the above shows $\pi(f)=\pi_{1}(F)$, where $F(\bar{g})=\int_{H} f(g h) d h, F \in L_{1}(G / H)$. Therefore $\pi(f)$ is compact and $G$ is CCR.

4. Nilpotent radical and traceable groups. If $H$ and $K$ are subgroups of a group $G$, we denote $[H, K]=$ the subgroup of $G$ generated by $\left\{x y x^{-1} y^{-1}: x \in H, y \in K\right\}$. Set $G_{1}=G^{1}=[G, G], G_{n}=\left[G_{n-1}, G_{n-1}\right]$, and $G^{n}=\left[G, G^{n-1}\right] . \quad G$ is called solvable (respectively nilpotent) if $G_{n}$ 
(respectively $G^{n}$ ) is trivial for some finite $n \geqq 1$. If $G$ is a topological group, it is well-known (and easily seen) that a subgroup $H$ is solvable (respectively nilpotent) $\Leftrightarrow \bar{H}$ is solvable (respectively nilpotent) see e.g. [15, Theorem 1.10].

DEFINITION. Let $G$ be a locally compact almost connected group. The radical $R=R(G)$ is the maximal connected solvable normal subgroup of $G$. The group $R$ does in fact exist [11, Theorem 15] and it is clearly closed. Also $R(G)=R\left(G_{0}\right) . \quad G$ is called semisimple if $R(G)$ is trivial.

Our next result is a generalization of a theorem due to Dixmier.

Theorem 4.1. Let $G$ be a locally compat almost connected group. Suppose that $R(G)$ is nilpotent. Then $G$ is type $I$.

Proof. Since $G / G_{0}$ is compact, it suffices [12, Theorem 1] to show that $G_{0}$ is type I. Also $R(G)=R\left(G_{0}\right) \Longrightarrow$ it is no loss of generality to assume that $G$ itself is connected. Then $G / R(G)$ is semisimple and $R(G)$ is the unique connected normal solvable subgroup $S \subseteq G$ such that $G / S$ is semisimple.

Now by Theorem 3.2, it suffices to show that for an arbitrary $H \in \mathscr{L}(G), G / H$ is type $I$. So fix $H \in \mathscr{L}(G), j=j_{H}$. Claim: $j(R(G))=$ $R(G / H)$. Indeed, $j(R(G))$ is a connected normal subgroup of $G / H$. In addition, a homomorphic image of a solvable group is again solvable. Therefore $j(R(G)) \subseteq R(G / H)$. But $j(R(G))=R(G) H / H$; hence the compactness of $H$ insures that $j(R(G))$ is closed in $G / H$. Denote $R_{1}=$ $j(R(G))$. Then $(G / H) / R_{1} \cong G / j^{-1}\left(R_{1}\right) \cong[G / R(G)] /\left[j^{-1}\left(R_{1}\right) / R(G)\right]$. But $G / R(G)$ is semisimple; and a factor group of a semisimple group is semisimple [15, Theorem 3.7]. Therefore $(G / H) / R_{1}$ is semisimple. It follows that $R_{1}=R(G / H)$.

A homomorphic image of a nilpotent group is also nilpotent. Therefore $R(G / H)$ must be nilpotent. But whenever a connected Lie group has nilpotent radical, the group must be type I [5, Prop. 2.3]. Therefore $G / H$ is type I.

Two special cases in which $R$ is nilpotent are: (1) $G$ is nilpotent itself, and (2) $G$ is semisimple. We can say somewhat more in these cases, but first we formulate some general notions. Let $G$ be almost connected, $H \in \mathscr{C}(G)$. Then $\mathscr{D}(G / H)$ is injected into $C_{0}(G)$ via $\omega_{H}$ : $\mathscr{D}(G / H) \rightarrow C_{0}(G),\left(\omega_{H} F\right)(g)=F\left(j_{H}(g)\right), g \in G$. The Schwartz-Bruhat space $\mathscr{D}(G)$ is the inductive limit $\mathscr{D}(G)=\lim _{\rightarrow I I \in \mathscr{S}}\left(\mathscr{D}(G / H), \omega_{H I}\right)$ (see $[1$, p. 45]). More precisely, $\mathscr{D}(G)$ is the linear span of the subspaces $\omega_{H}(\mathscr{D}(G / H))$ with the strongest topology making all the maps $\omega_{H}$ continuous. 
Definition. $G$ is called traceable if for every $\pi \in \operatorname{Irr}(G)$ and every $f \in \mathscr{D}(G)$, the operator $\pi(f)=\int_{G} f(g) \pi(g) d g$ is trace class and $f \rightarrow \operatorname{Tr} \pi(f)$ is a distribution. The distribution $\theta_{\pi}: f \rightarrow \operatorname{Tr} \pi(f)$ is called the character of $\pi$. It depends only on the class of $\pi$ in $\widehat{G}$.

Note $G$ traceable implies $G$ is CCR. Indeed $\pi(\mathscr{D}(G)) \subseteq$ trace class operators $\subseteq$ compact operators. But $\mathscr{D}(G)$ is dense in $L_{1}(G)$,

$$
\|\pi(f)\| \leqq\|f\|_{1}
$$

and the compact operators are closed in the operator norm $\Rightarrow \pi\left(L_{1}(G)\right) \subseteq$ compact operators. Traceable appears to be a stronger property than CCR, but experience has shown that many CCR groups are indeed traceable.

THEOREM 4.2. Let $G$ be a connected locally compact group. Suppose that $G$ is either semisimple or nilpotent. Then $G$ is traceable. If $G$ is semisimple and $\pi \in \hat{G}$, then $\theta_{\pi}$ is actually a locally integrable function, i.e., $\operatorname{Tr} \pi(f)=\int_{G} f(g) \psi_{\pi}(g) d g, f \in \mathscr{D}(G)$, where $\psi_{\pi} \in L_{1}^{\mathrm{loc}}(G)$.

Proof. Suppose first that $G$ is a connected Lie group. If $G$ is semisimple, then all claims of the theorem are known and due to Harish-Chandra [8,9]. On the other hand if $G$ is nilpotent, then Dixmier [2, p. 78] has established the traceability of $G$.

In general now, let $G$ be connected and either semisimple or nilpotent. Let $\pi \in \operatorname{Irr}(G)$. By Theorem 2.1 there exists $H_{1} \in \mathscr{L}(G)$ such that $\left.\pi\right|_{H_{1}}=I$. Next let $f \in \mathscr{D}(G)$. Then there exists $H_{2} \in \mathscr{L}(G)$ such that $f$ is $H_{2}$-invariant and $f=\omega_{H}(\bar{f}), \bar{f} \in \mathscr{D}\left(G / H_{2}\right)$. Set $H=H_{1} \cap$ $H_{2} \in \mathscr{L}(G)$. Writing $\bar{g}=j_{H}(g), \bar{f}(\bar{g})=f(g), \pi_{H}(\bar{g})=\pi(g)$, we compute $\pi(f)=\int_{G} f(g) \pi(g) d g=\int_{G / H} \bar{f}(\bar{g}) \pi_{H}(\bar{g}) d \bar{g}$. But the factor group $G / H$ must be semisimple or nilpotent according as $G$ is. Also $\bar{f} \in \mathscr{D}(G / H)$. Therefore by the Lie group case $\pi(f)$ is trace class. Moreover, it is clear that $\theta_{\pi}(f)=\operatorname{Tr} \pi(f)$ is independent of the choice of $H \in \mathscr{L}(G)$ such that $\left.\pi\right|_{H}=I$ and $f$ is $H$-invariant. It remains to show that $f \rightarrow$ $\theta_{\pi}(f), \mathscr{D}(G) \rightarrow C$, is continuous.

Since $\mathscr{X}(G)=\lim _{\rightarrow}\left(\mathscr{D}(G / H), \omega_{H}\right)$, it is enough to show $\theta_{\pi} \circ \omega_{I I}$ is continuous for all $H \in \mathscr{L}(G)$. In fact it suffices to prove that $\theta_{\pi} \circ \omega_{H}$ is continuous for all sufficiently small $H$. Therefore we fix $H \in \mathscr{L}$ such that $\left.\pi\right|_{H}=I$, and show that $\theta_{\pi} \circ \omega_{K}$ is continuous for all $K \in \mathscr{L}$, $K \subseteq H$. But it is a formality to check that $\theta_{\pi^{\circ}} \omega_{K}=\theta_{\pi_{K}}, \pi_{K}\left(j_{K}(g)\right)=$ $\pi(g)$. Therefore (again by the Lie group case), $\theta_{\pi_{K}}$ is continuous; and so 
we obtain the fact that $\theta_{\pi}$ is a distribution.

Finally, suppose $G$ is semisimple. Choosing $H \in \mathscr{L}(G)$ such that $\left.\pi\right|_{H}=I, \pi=\hat{j}_{H} \pi_{H}$, we define $\psi_{\pi}(g)=\psi_{\pi_{H}}\left(j_{H}(g)\right), g \in G$. It is straightforward to check that the function $\psi_{\pi}$ is independent of the choice of $H$ with these properties. Certainly $\psi_{\pi} \in L_{1}^{\text {loc }}(G)$. Moreover for any $f \in \mathscr{D}(G)$, we can choose $K \in \mathscr{L}$ such that $K \subseteq H$ and $f$ is $K$-invariant. Then $\operatorname{Tr} \pi(f)=\operatorname{Tr} \pi_{K}\left(f_{K}\right)=\int_{G / K} f_{K}(\bar{g}) \psi_{\pi}(\bar{g}) d \bar{g}=\int_{G} f(g) \psi_{\pi}(g) d g$. This completes the proof.

REMARK. The same techniques as in the above proof show that to extend Theorem 4.2 to the almost connected case, one need only consider a Lie group $G$ with finitely many components, [G: $\left.G_{0}\right]<\infty$. Using the fact that an irreducible representation of $G$ breaks up into a direct sum of at most $\left[G: G_{0}\right]$ irreducible representations when restricted to $G_{0}$ (a fact which can be justified by using the Mackey machine for normal subgroups), one can show that $\pi \in \operatorname{Irr}(G), f \in$ $\mathscr{D}(G) \Rightarrow \pi(f)$ is trace class. I have not been able to prove that $f \rightarrow$ $\operatorname{Tr} \pi(f)$ is continuous nor that in the semisimple case the character is a function. More generally, I don't know if a finite extension of a traceable group need be traceable.

5. Topology and measure. In this section we give topological and measure-theoretic interpretations of equation (1.3).

Suppose that $X$ is a locally compact (but not necessarily Hausdorff) topological space. As usual the Borel sets $\mathscr{B}(X)$ constitute the smallest $\sigma$-algebra of subsets of $X$ containing all open sets. It is possible for a compact subset of $X$ to be non-Borel. However, we assume that every point of $X$ has a neighborhood basis of compact Borel sets. By a positive Radon measure on $X$ we mean a map $\mu: \mathscr{B}(X) \rightarrow[0, \infty]$ having the properties: (i) $\mu$ is countably additive, (ii) $\mu(C)<\infty$ for every compact Borel set $C$, (iii) for every $Y \in \mathscr{B}(X)$, we have $\mu(Y)=\sup \{\mu(C): C \subseteq Y, C$ compact Borel $\}$.

Example. $G=$ unimodular type I locally compact group, $X=\hat{G}$, $\mu=$ the Plancherel measure $\mu_{G}$ (see [3]).

LemMa 5.1. Let $X$ be a locally compact space. Suppose that $\left\{X_{\alpha}\right\}_{\alpha \in \Omega}$ is an increasing net of open subsets of $X$ whose union equals $X$. Assume that each $X_{\alpha}$ carries a positive Radon measure $\mu_{\alpha}$ such that whenever $X_{\alpha} \subseteq X_{\beta}$, we have $\left.\mu_{\beta}\right|_{X_{\alpha}}=\mu_{\alpha}$. Then there exists a unique positive Radon measure $\mu$ on $X$ such that $\left.\mu\right|_{X_{\alpha}}=\mu_{\alpha}, \alpha \in \Omega$.

Proof. Let $Y \in \mathscr{B}(X)$. For any $C \in \mathscr{B}(X), C \subseteq Y, C$ compact, 
it must be true that $C \subseteq X_{\alpha}$ some $\alpha$. Set $\mu(Y)=\sup _{C}\left\{\mu_{\alpha}(C)\right\}$. It is a relatively straightforward mattter to check that $\mu$ is the desired measure (see [3, Lemma 16]).

We call $\mu$ the inductive limit of the $\mu_{\alpha}$ and write $\mu=\lim _{\rightarrow \alpha \in \Omega} \mu_{\alpha}$.

LEMma 5.2. Let $G$ be a locally compact group, $H \subseteq G$ a compact normal subgroup. As usual $j_{H}: G \rightarrow G / H$ is the canonical projection, and $\hat{j}_{H}: \widehat{G / H} \rightarrow \hat{G}$ is the adjoint map, $\hat{G}_{H}=\hat{j}_{H}(\widehat{G / H})$.

(i) $\widehat{G}_{H}$ is an open-closed subset of $\hat{G}$, and $\hat{j}_{H}: \widehat{G / H} \rightarrow \widehat{G}_{H}$ is a homeomorphism.

(ii) Suppose in addition that $G$ is unimodular and type $I$. Then $G / H$ has those properties also, and $\left.\mu_{G}\right|_{\hat{G}_{H}}=\mu_{G / H}$.

Proof. Let us first briefly recall how the topology on $\hat{G}$ may be defined (see [6]). If $\pi \in \hat{G}$ and $\mathscr{S} \leqq \hat{G}$ is a subset, then $\pi \in \overline{\mathscr{S}}$ if and only if every continuous positive-definite function associated to $\pi$ is a uniform-on-compacta limit of continuous positive-definite functions associated to $\mathscr{S}$; more precisely, given $\xi \in \mathscr{X}(\pi), M \cong G$ compact subset, $\varepsilon>0$, then there exists $\sigma \in \mathscr{S}, \eta \in \mathscr{C}(\sigma)$ such that

$$
|(\pi(g) \xi, \xi)-(\sigma(g) \eta, \eta)|<\varepsilon, \text { all } g \in M \text {. }
$$

(i) The fact that $\hat{j}_{H}: \widehat{G / H} \rightarrow \widehat{G}_{I I}$ is a homeomorphism is wellknown and requires only that $H$ is a closed normal subgroup. The same is true of the fact that $\widehat{G}_{H}$ is closed, but I include a proof for illustrative purposes. Of course $\hat{G}_{I I}=\left\{\pi \in \hat{G}:\left.\pi\right|_{H}=I\right\}$. Suppose $\pi \epsilon$ $\left.\widehat{G}_{H}\right)^{-}$. Let $h \in H$ be arbitrary. Then we can find a compact subset $M \subseteq G$ containing $e, h$. Let $\xi$ be any vector in $\mathscr{C}(\pi)$ and set $f(g)=$ $(\pi(g) \xi, \xi)$. Finally let $\varepsilon>0$. Then there exists $\sigma \in \widehat{G}_{I I}$ and $\eta \in \mathscr{X}(\sigma)$ such that $|f(g)-(\sigma(g) \eta, \eta)|<\varepsilon, g \in M$. Since $\sigma \in \hat{G}_{H},\left.\sigma\right|_{I I}=I$. Setting $g=e$ and then $g=h$, we obtain $\left|f(h)-\|\xi\|^{2}\right|<2 \varepsilon$. Since $\varepsilon$ is arbitrary, $f(h)=\|\xi\|^{2}$, i.e., $(\pi(h) \xi, \xi)=(\xi, \xi), \xi \in \mathscr{L}(\pi)$. Therefore $\pi(h)$ is both unitary and positive $\Rightarrow \pi(h)=I$. Since $h \in H$ was arbitrary, $\left.\pi\right|_{H}=I \Rightarrow \pi \in \widehat{G}_{H}$. Using similar arguments, it is easy to show $\mathscr{S} \leqq$ $\widehat{G / H}$ is closed $\Leftrightarrow \hat{j}_{H}(\mathscr{S}) \subseteq \widehat{G}_{H}$ is closed. (We omit the details.)

We next prove that $\hat{G}_{I I}$ is also open. Unlike the previous situation, this requires that $H$ be a compact subgroup. We prove that $\mathscr{S}=\hat{G}-\widehat{G}_{H}=\left\{\pi \in \hat{G}:\left.\pi\right|_{I I} \neq I\right\}$ is closed. Let $\pi \in \overline{\mathscr{S}}$. Choose a nonzero vector $\xi \in \mathscr{H}(\pi)$ and select $M=H$ itself. Let $\varepsilon>0$. Then there exists $\sigma \in \mathscr{S}, \eta \in \mathscr{H}(\sigma)$ such that $|(\pi(h) \xi, \xi)-(\sigma(h) \eta, \eta)|<\varepsilon, h \in H$. Suppose that $\pi \notin \mathscr{S}$. Then $\left.\pi\right|_{H}=I \Rightarrow\left|\|\xi\|^{2}-(\sigma(h) \eta, \eta)\right|<\varepsilon, h \in H$. We claim that the operator $\int_{H} \sigma(h) d h$ is the zero operator. Postponing 
the proof of that momentarily, we see that

$$
0<\|\xi\|^{2}=\left|\int_{H}\left[\|\xi\|^{2}-(\sigma(h) \eta, \eta)\right] d h\right| \leqq \int_{H}\|\xi\|^{2}-(\sigma(h) \eta, \eta) \mid d h<\varepsilon .
$$

Since $\varepsilon$ is arbitrary, this is a contradiction.

It remains to establish the claim. Since we will have use for it again later, we state it as a separate

Lemma 5.3. If $\sigma \in \hat{G}$ and $\left.\sigma\right|_{H} \neq I$, then $\int_{H} \sigma(h) d h=0$.

Proof. If $\left.\sigma\right|_{H}$ is irreducible, then this is an immediate consequence of the orthogonality relations for the compact group $H\left(\left.\sigma\right|_{H} \neq\right.$ $\left.I \Longrightarrow \sigma\right|_{H}$ is orthogonal to the identity representation). Otherwise $\left.\sigma\right|_{I I}=$ $\bigoplus_{\beta} \sigma_{\beta}, \sigma_{\beta} \in \hat{H}$. Since $\int \sigma(h) d h=\bigoplus_{\beta} \int \sigma_{\beta}(h) d h$, it's enough to show that the identity representation does not occur as an irreducible constituent of $\left.\sigma\right|_{H}$. But if there is $\xi \in \mathscr{L}(\sigma), \xi \neq 0, \sigma(h) \xi=\xi, h \in H$, then

$$
\sigma(h) \sigma(g) \xi=\sigma(g) \sigma\left(g^{-1} h g\right) \xi=\sigma(g) \xi, g \in G, h \in H .
$$

Since $\sigma$ is an irreducible representation of $G, \xi$ must be a cyclic vector $\Longrightarrow \sigma(h) \eta=\eta$, all $\eta \in \mathscr{H}(\sigma), h \in H$, a contradiction to the original assumption $\left.\sigma\right|_{H} \neq I$. This completes the proof of Lemma 5.3 and part (i) of Lemma 5.2.

(ii) If $G$ is type I, then so is $G / H$ (the argument is a duplication of the first paragraph in the proof of Theorem 3.2). Also $G$ unimodular and $H$ compact normal $\Rightarrow G / H$ is unimodular. Finally we show that $\mu_{G} \mid \hat{G}_{I I}=\mu_{G / I I}$. Let $F \in L_{1}(G / H) \cap L_{2}(G / H)$, and set $f(g)=F(\bar{g}), g \in G$, $\bar{g}=j_{H}(g)$. Certainly $f \in L_{1}(G) \cap L_{2}(G)$, and $f$ is right $H$-invariant. Suppose $\pi \in \hat{G}$ and $\xi, \eta \in \mathscr{H}(\pi)$. Then

$$
\begin{aligned}
(\pi(f) \xi, \eta) & =\int_{G} f(g)(\pi(g) \xi, \eta) d g \\
& =\int_{G / H} \int_{H} f(g h)(\pi(g h) \xi, \eta) d h d \bar{g} \\
& =\int_{G / H} F(\bar{g}) d \bar{g} \int_{I I}\left(\pi(h) \xi, \pi\left(g^{-1}\right) \eta\right) d h .
\end{aligned}
$$

Therefore if $\pi \notin \widehat{G}_{I I}$, i.e., $\left.\pi\right|_{H} \neq I$, it follows from Lemma 5.3 that $\pi(f)=0$. On the other hand if $\pi \in \widehat{G}_{I I}, \pi=\hat{j}_{H I} \pi_{H}$, then $\pi(f)=\pi_{H}(F)$.

We apply the Plancherel formula (1.1) to $G / H$ and then to $G$. First

$$
\int_{G / H}|F(\bar{g})|^{2} d \bar{g}=\int_{\widehat{G / H}} \operatorname{Tr}\left[\pi_{H}(F) * \pi_{H}(F)\right] d \mu_{G / H}(\pi) .
$$

Then we also have 


$$
\begin{aligned}
\int_{G / H}|F(\bar{g})|^{2} d \bar{g}= & \int_{G}|f(g)|^{2} d g=\int_{\hat{G}} \operatorname{Tr}\left[\pi(f)^{*} \pi(f)\right] d \mu_{G}(\pi) \\
= & \int_{\hat{G}_{H}} \operatorname{Tr}\left[\pi(f)^{*} \pi(f)\right] d \mu_{G}(\pi) \\
& +\int_{\hat{a}_{-} \hat{G}_{H}} \operatorname{Tr}\left[\pi(f)^{*} \pi(f)\right] d \mu_{G}(\pi) \\
= & \int_{\hat{G}_{H}} \operatorname{Tr}\left[\pi_{H}(F)^{*} \pi_{H}(F)\right] d \mu_{G}(\pi) .
\end{aligned}
$$

Since $F \in L_{1}(G / H) \cap L_{2}(G / H)$ was arbitrary, it follows from the uniqueness of the Plancherel measure that $\left.\mu_{G}\right|_{\hat{G}_{H}}=\mu_{G \mid H}$.

We can now state the main result of this section.

THEOREM 5.4. Let $G$ be a locally compact almost connected group.

(i) $\hat{G}=\lim _{H \in \mathscr{L}} \widehat{G / H}$ topologically in the sense that the maps $\hat{j}_{I I}: \widehat{G / H} \rightarrow \widehat{G} \overrightarrow{\text { are }}$ homeomorphisms onto open-closed subsets, the collection of which forms an increasing net whose union is $\hat{G}$.

(ii) Suppose in addition that $G$ is unimodular and type $I$. Then $\mu_{G}=\lim _{H \in \mathscr{L}} \mu_{G J H}$.

Proof. (i) This follows immediately from Lemma 5.2 and Theorem 2.1.

(ii) Suppose that $K \cong H$, both in $\mathscr{L}(G)$, so that $\widehat{G}_{H} \subseteq \widehat{G}_{K}$. Then $G / H \cong(G / K) /(H / K)$. By Lemma 5.2, $\widehat{G / H}$ (which is homeomorphic to $\hat{G}_{I I}$ ) is also homeomorphic to an open subset $\mathscr{Q}=\widehat{G / H}_{H / K}$ of $\widehat{G / K}$ (the latter being homeomorphic to $\left.\widehat{G}_{K}\right)$. In addition $\left.\mu_{G / K}\right|_{\|}=\mu_{G / I I}$. Therefore we may apply Lemma 5.1. The conclusion is that there is a unique positive Radon measure $\mu=\lim _{I I \in \mathscr{S}} \mu_{G \mid H}$ such that $\left.\mu\right|_{\hat{\sigma}_{H}}=\mu_{G \mid I I}$, $H \in \mathscr{L}$. But, again by Lemma $5.2, \mu_{G}$ is a positive Radon measure on $\hat{G}$ such that $\left.\mu_{G}\right|_{\hat{G}_{H}}=\mu_{G / H}, H \in \mathscr{L}$. Hence $\mu=\mu_{G}$ and we are done.

REMARK. It is easily checked that (i) forces $\hat{G}$ to be the ordinary topological inductive limit of the $\widehat{G / H}$; that is, the dual topology on $\hat{G}$ is the strongest which makes all the maps $\hat{j}_{H}: \widehat{G / H} \rightarrow \widehat{G}$ continuous.

6. Appendix-semisimple groups. In $\S 5$ we showed how to obtain the Plancherel measure for an almost connected group as an inductive limit of Plancherel measures for Lie groups. We indicate here how, for semisimple groups, the representation theory and Plancherel measure can be constructed in terms of the "parameters" of the group itself.

Lemma 6.1. Let $G$ be a connected semisimple group. Suppose 
that $\Omega=$ the maximal compact normal subgroup of $G$. Let $Z=$ the centralizer of $\Omega$ in $G$ and $G_{1}=Z_{0}$ Then:

(i) $G=G_{1} \Omega$;

(ii) $G_{1}$ and $\Omega$ commute;

(iii) $G_{1}$ is a closed normal subgroup of $G$ which is connected, semisimple, and finite-dimensional.

Proof. It is known that $G$ has maximal compact subgroups and that they are all conjugate [11, Theorem 13]. Therefore the intersection of all of them yields the maximal compact normal subgroup $\Omega$. It is clear that $\Omega$ is the largest element of $\mathscr{L}(G)$.

(i) is an immediate consequence of a theorem of Iwasawa (see, e.g., [15, Theorem 1.4]). (ii) is obvious. In (iii), the fact that $G_{1}$ is closed, normal and connected is clear. Moreover, since any normal subgroup of $G_{1}$ is normal in $G$, it follows that $G_{1}$ is semisimple. Finally, $H=G_{1} \cap \Omega$ is abelian and normal in $G$; therefore it is totallydisconnected (zero-dimensional). Moreover $G / \Omega=G_{1} \Omega / \Omega \cong G_{1} / H$ is a Lie group. This is, $G_{1}$ is finite-dimensional. Actually, it's not hard to show that properties (i)-(iii) characterize $G_{1}$ uniquely.

Set $K=\left\{(h, h): h \in H=G_{1} \cap \Omega\right\}$. Then $G \cong\left(G_{1} \times \Omega\right) / K$ and $\hat{G} \cong$ $\left(G_{1} \times \Omega\right)_{K} \cong\left(\hat{G}_{1} \times \widehat{\Omega}\right)_{K}$; so it is reasonable to restrict our attention to the finite-dimensional case.

Let $G$ be connected, semisimple, and finite-dimensional. Then there is $H \subseteq G$, a compact, totally-disconnected normal subgroup such that $G / H$ is a Lie group. Since $G$ is connected, $H \subseteq Z_{G}=\operatorname{Center}(G)$. Therefore $G / Z_{G}$ is also a Lie group. Let $\mathfrak{g}=$ its Lie algebra. One checks easily that $G / Z_{G}$ is a connected semisimple Lie group with no center; therefore $G / Z_{G} \cong \operatorname{Int} \mathrm{g}=$ the adjoint group of $\mathrm{g}$. The canonical projection $G \rightarrow G / Z_{G}$ may thus be considered to be the "adjoint representation" Ad: $G \rightarrow$ Intg. We can now develop the structure theory of $G$ exactly as in the Lie group case. For example, let $\mathfrak{y} \cong \mathfrak{g}$ be a Cartan subalgebra. Then $C=$ the centralizer of $\mathfrak{y}$ in $G=\{g \in$ $G$ : Ad $g(X)=X, X \in \mathfrak{1}\}$ is called a Cartan subgroup. Let us now make the additional assumption that $Z_{G}$ is compact. This is analogous to the usual assumption of finite center in the Lie group case. One also needs to make a technical assumption corresponding to Harish-Chanda's concept of acceptability [9, p. 484], but we shall not elaborate on that here. Then we can carry through Harish-Chandra's entire theory for semisimple Lie groups. Briefly, it goes as follows.

Suppose $\mathfrak{g}=\mathfrak{f}+\mathfrak{q}$ is a Cartan decomposition. Then there is a maximal compact subgroup $K$ of $G$ such that $\operatorname{Ad}(K)$ has $\mathfrak{f}$ as Lie algebra. $G$ has a discrete series (i.e., a family of square-integrable irreducible unitary representations) if and only if there is a Cartan 
subalgebra $\mathfrak{y} \subseteq \mathfrak{f}$. In that case, the corresponding Cartan subgroup $C \subseteq K$ is compact, connected, abelian, and finite-dimensional. The discrete series is parameterized roughly by the charaters of $C . \hat{C}$ of course is a discrete space, but actually there is still an inductive limit buried in it. In fact $C$, being a finite-dimensional, compact, connected, abelian group, is a projective limit of tori (of bounded dimension). Taking the dual, we see that $\hat{C}$ is an inductive limit of lattice groups (again of bounded dimension).

We now describe how to obtain the continuous (or principal) series. A subgroup $P \subseteq G$ is called parabolic if it is closed, $\mathfrak{p}=$ Lie algebra of $\mathrm{Ad}(P)$ is parabolic (i.e., its complexification contains a maximal solvable subalgebra of the complexification of $\mathrm{g}$ ), and $P=$ normalizer $(\mathfrak{p})=\{g \in G: \operatorname{Ad} g(X) \in \mathfrak{p}, X \in \mathfrak{p}\}$. Exactly as in the Lie case one obtains a Langlands decomposition $P=M A N$ where $N$ is a connected, simplyconnected nilpotent group, $A$ is a vector group, and $M$ is a finitedimensional reductive (i.e., $\mathfrak{m}=$ Lie algebra of $\operatorname{Ad}(M)$ is reductive) group. $P$ is called cuspidal if $M$ contains a compact Cartan subgroup $B$ (i.e., if there is $\mathfrak{b} \subseteq \mathfrak{m}$, a Cartan subalgebra such that $B=\{m \in$ $M:$ Ad $m(X)=X, X \in \mathfrak{b}\}$ is compact). In that case $C=B A$ is a Cartan subgroup of $G$; in fact, $\mathfrak{y}=$ Lie algebra of $\operatorname{Ad}(C)$ is a Cartan subalgebra of $\mathfrak{g}$ and $C=$ centralizer (y).

Two parabolics $P_{1}, P_{2}$ are called associate if the corresponding Cartan subalgebras $\mathfrak{y}_{1}, \mathfrak{y}_{2}$ are conjugate (under $G$ or Int $\mathfrak{g}$ ). The number of associativity classes is finite, and for each we get a distinct family of continuous series representations as follows: take $\lambda$ in the discrete series of $M, \nu \in \hat{A}$, and form $\operatorname{Ind}_{P}^{F} \gamma, \gamma(\operatorname{man})=\nu(a) \lambda(m)$. The discrete series of the reductive group $M$ is somewhat complicated since $M$ is disconnected in general. It can be worked out using arguments similar to those in [13, §4], where the corresponding problem for Lie groups was solved. In any event, exactly as in the Lie case, the Plancherel measure lives on these series and can be computed quite explicitly. The precise result is in complete analogy with Harish-Chandra's formula for semisimple Lie groups [10, p. 545].

\section{REFERENCES}

1. F. Bruhat, Distributions sur un groupe localement compact et applications à l'étude des représentations des groupes p-adiques, Bull. Soc. Math. France, 89 (1961), 43-75.

2. J. Dixmier, Sur les représentations unitaries des groupes de Lie nilpotents $V$, Bull. Soc. Math. France, 87 (1959), 65-79.

3. - Traces sur les $C^{*}$-algèbres, Ann. Inst. Fourier, 13 (1963), 219-262.

4. - Les $C^{*}$-Algèbres et leurs Représentations, Gauthier-Villars, Paris, 1964.

5. - Sur la représentation régulière d'un groupe localment compact connexe, Ann. Scient. École Norm. Sup., 2 (1969), 423-436. 
6. J. Fell, The dual spaces of $C^{*}$-algebras, Trans. Amer. Math. Soc., 94 (1960), 365403.

7. - A new proof that nilpotent groups are CCR, Proc. Amer. Math. Soc., 13 (1962), 93-99.

8. Harish-Chandra, Representations of semisimple Lie groups III, Trans. Amer. Math. Soc., 76 (1954), 234-253.

9. - Invariant eigendistributions on a semisimple Lie group, Trans. Amer. Math. Soc., 119 (1965), 457-508.

10. - Harmonic analysis on semisimple Lie groups, Bull. Amer. Math. Soc., 76 (1970), 529-551.

11. K. Iwasawa, On some types of topological groups, Ann. of Math., 50 (1949), 507558.

12. R. Kallman, Certain topological groups are type I, Bull. Amer. Math. Soc., 76 (1970), 404-406.

13. R. Lipsman, On the characters and equivalence of continuous series representations, J. Math. Soc. Japan, 23, No. 3 (1971), 452-480.

14. D. Montgomery and L. Zippin, Topological Transformation Groups, Interscience, New York, 1955.

15. N. Rickert, Some properties of locally compact groups, J. Australian Math. Soc., 7 (1967), 433-454.

16. S. Sakai, On a characterization of type $I C^{*}$-algebras, Conference on functional analysis at the University of California, Irvine, B. Gelbaum, ed., Thompson Book Co., Washington, D. C., 1967.

Received May 21, 1971.

UNIVERSITY OF MARYLAND 



\section{PACIFIC JOURNAL OF MATHEMATICS}

\section{EDITORS}

\author{
H. SAMELSON \\ Stanford University \\ Stanford, California 94305 \\ C. R. HOBBY \\ University of Washington \\ Seattle, Washington 98105
}

\section{J. DuGundJI}

Department of Mathematics University of Southern California Los Angeles, California 90007

RICHARD ARENS

University of California

Los Angeles, California 90024

\section{ASSOCIATE EDITORS}
E. F. BECKENBACH
B. H. NeumanN
F. WOLF
K. YOSHIDA

\section{SUPPORTING INSTITUTIONS}

\author{
UNIVERSITY OF BRITISH COLUMBIA \\ CALIFORNIA INSTITUTE OF TECHNOLOGY \\ UNIVERSITY OF CALIFORNIA \\ MONTANA STATE UNIVERSITY \\ UNIVERSITY OF NEVADA \\ NEW MEXICO STATE UNIVERSITY \\ OREGON STATE UNIVERSITY \\ UNIVERSITY OF OREGON \\ OSAKA UNIVERSITY
}

\author{
UNIVERSITY OF SOUTHERN CALIFORNIA \\ STANFORD UNIVERSITY \\ UNIVERSITY OF TOKYO \\ UNIVERSITY OF UTAH \\ WASHINGTON STATE UNIVERSITY \\ UNIVERSITY OF WASHINGTON \\ AMERICAN MATHEMATICAL SOCIETY \\ NAVAL WEAPONS CENTER
}

The Supporting Institutions listed above contribute to the cost of publication of this Journal, but they are not owners or publishers and have no responsibility for its content or policies.

Mathematical papers intended for publication in the Pacific Journal of Mathematics should be in typed form or offset-reproduced, (not dittoed), double spaced with large margins. Underline Greek letters in red, German in green, and script in blue. The first paragraph or two must be capable of being used separately as a synopsis of the entire paper. The editorial "we" must not be used in the synopsis, and items of the bibliography should not be cited there unless absolutely necessary, in which case they must be identified by author and Journal, rather than by item number. Manuscripts, in duplicate if possible, may be sent to any one of the four editors. Please classify according to the scheme of Math. Rev. Index to Vol. 39. All other communications to the editors should be addressed to the managing editor, Richard Arens, University of California, Los Angeles, California, 90024.

50 reprints are provided free for each article; additional copies may be obtained at cost in multiples of 50 .

The Pacific Journal of Mathematics is published monthly. Effective with Volume 16 the price per volume (3 numbers) is $\$ 8.00$; single issues, $\$ 3.00$. Special price for current issues to individual faculty members of supporting institutions and to individual members of the American Mathematical Society: $\$ 4.00$ per volume; single issues $\$ 1.50$. Back numbers are available.

Subscriptions, orders for back numbers, and changes of address should be sent to Pacific Journal of Mathematics, 103 Highland Boulevard, Berkeley, California, 94708.

PUBLISHED BY PACIFIC JOURNAL OF MATHEMATICS, A NON-PROFIT CORPORATION

Printed at Kokusai Bunken Insatsusha (International Academic Printing Co., Ltd.), 270, 3-chome Totsuka-cho, Shinjuku-ku, Tokyo 160, Japan. 


\section{Pacific Journal of Mathematics}

\section{Vol. 42, No. $2 \quad$ February, 1972}

Stephen Richard Bernfeld, The extendability of solutions of perturbed scalar differential equations ................................. 277

James Edwin Brink, Inequalities involving $f_{-} p$ and $f^{(n)}{ }_{q}$ for $f$ with $n$

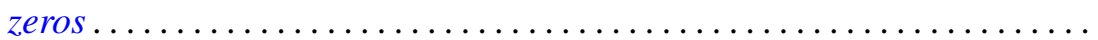

Orrin Frink and Robert S. Smith, On the distributivity of the lattice of filters of a groupoid

Donald Goldsmith, On the density of certain cohesive basic sequences .... 323

Charles Lemuel Hagopian, Planar images of decomposable continua . . . . . 329

W. N. Hudson, A decomposition theorem for biadditive processes ........ 333

W. N. Hudson, Continuity of sample functions of biadditive processes......

Masako Izumi and Shin-ichi Izumi, Integrability of trigonometric series.

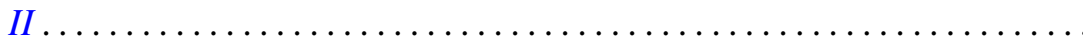

H. M. Ko, Fixed point theorems for point-to-set mappings and the set of

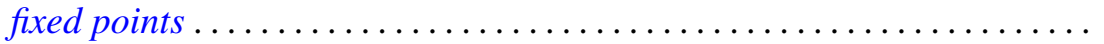

Gregers Louis Krabbe, An algebra of generalized functions on an open interval: two-sided operational calculus ...

Thomas Latimer Kriete, III, Complete non-selfadjointness of almost selfadjoint operators.................................

Shiva Narain Lal and Siya Ram, On the absolute Hausdorff summability of a Fourier series .

Ronald Leslie Lipsman, Representation theory of almost connected groups...

James R. McLaughlin, Integrated orthonormal series.... . .

H. Minc, On permanents of circulants.

Akihiro Okuyama, On a generalization of $\Sigma$-spaces.....

Norberto Salinas, Invariant subspaces and operators of class $(S)$

James D. Stafney, The spectrum of certain lower triangular matrices as operators on the $l_{p}$ spaces .......................

Arne Stray, Interpolation by analytic functions

$\mathrm{Li} \mathrm{Pi} \mathrm{Su}$, Rings of analytic functions on any subset of the complex plane.

R. J. Tondra, A property of manifolds compactly equivalent to compact manifolds.... 